\title{
COMUnicación
}

\section{Uso de Plasma Seminal en la Criopreservación de Espermatozoides Epididimarios de Equinos}

\author{
Use of Seminal Plasma in Equine Epididymal Spermatozoa Cryopreservation \\ H. Mauricio Gonzales Molfino ${ }^{1,2,3}$, Carolina Rodríguez ${ }^{1}$, Anderson Oropeza ${ }^{1}$, \\ Yat Sen Wong ${ }^{1}$, José Llanos ${ }^{1}$, Hugo Gonzales Figueroa ${ }^{1}$
}

\section{Resumen}

\begin{abstract}
Se utilizó plasma seminal equino en un dilutor de lactosa-EDTA para la criopreservación de espermatozoides epididimarios de equinos. Se trabajó con 12 pares de testículos de caballos beneficiados. Se separaron los epidídimos y se utilizó la técnica de lavado retrógrado para obtener los espermatozoides, inyectando $10 \mathrm{ml}$ del dilutor lactosa-EDTA por el conducto deferente. Se utilizaron las muestras con más de 30\% de motilidad progresiva. Las muestras fueron diluidas 1:1 con el diluyente lactosa-EDTA-plasma seminal y se envasó en pajillas de $0.5 \mathrm{ml}$ a una concentración $386.3 \times 10^{6}$, y fueron congeladas en nitrógeno líquido y almacenadas por 10 días. Los valores obtenidos para las muestras frescas y descongeladas fueron: motilidad progresiva: 43.3 y $16.4 \%(\mathrm{p}<0.05)$, viabilidad: 48.3 y $40.5 \%$, morfología normal: 67.1 y $56.5 \%$, e integridad de la membrana plasmática (HOS): 48.3 y $45.5 \%$.
\end{abstract}

Palabras clave: espermatozoides, criopreservación, plasma seminal, epidídimo, equinos

\section{Abstract}

Equine seminal plasma was used in a lactose-EDTA extender for cryopreservation of equine epididymal sperm. Twelve pairs of testicles of slaughtered horses were used. Epididymides were separated and washed applying the retrograde technique to obtain

\footnotetext{
${ }^{1}$ Laboratorio de Biotecnología y Fisiología Animal, Facultad de Ciencias Biológicas, Universidad Ricardo Palma, Lima, Perú

${ }^{2}$ Escuela de Medicina Veterinaria, Universidad Ricardo Palma, Lima, Perú

${ }^{3}$ E-mail: mauricio.gonzalesm@urp.pe
}

Recibido: 26 de junio de 2014

Aceptado para publicación: 28 de noviembre de 2014 
sperm by injecting $10 \mathrm{ml}$ of lactose-EDTA extender through the vas deferens. Samples with more than $30 \%$ motility were used. Samples were diluted 1: 1 with the lactose-EDTAextender seminal plasma and filled into $0.5 \mathrm{ml}$ straws at a concentration of $386.3 \times 10^{6}$, frozen in liquid nitrogen and stored for 10 days. The values for fresh and thawed samples were: motility: 43.3 and $16.4 \%(p<0.05)$, viability: 48.3 and $40.5 \%$, normal morphology: 67.1 and $56.5 \%$, and integrity of the plasma membrane (HOS): 48.3 and $45.5 \%$.

Key words: sperm, cryopreservation, plasma seminal, epididymis, equine

\section{INTRODUCCIÓN}

En la actualidad, la recuperación de espermatozoides y la preservación del epidídimo de un semental después de la castración, sea por decisión propia, lesión traumática, enfermedad grave o muerte inesperada, ha permitido la conservación genética gracias los avances en biotecnología reproductiva (James, 2004; Bruemmer, 2006). Se ha demostrado que espermatozoides recuperados de epidídimo son viables, incluso cuando se les mantiene a temperatura ambiente hasta por 24 horas, lo cual indica que incluso podrían ser utilizados en inseminación artificial (IA) (Papa et al., 2008); no obstante, cuando los testículos se almacenan a $4^{\circ} \mathrm{C}$, el esperma tiende a tener una mayor viabilidad (James, 2004).

En el equino, el lavado retrógrado es una técnica rápida y eficaz para la recuperación de espermatozoides de la cola de epidídimo, y de calidad similar a la obtenida en eyaculados recogidos con vagina artificial (Papa et al., 2008; Monteiro et al., 2011); asimismo, estos espermatozoides congelados demuestran ser fértiles (Cary et al., 2004). Otros estudios demuestran que espermatozoides de cola de epididimo obtenidos dentro de las 24 horas de la orquiectomía tienen similar fertilidad a los del esperma eyaculado (Armas et al., 2011; Monteiro et al., 2011).

La criopreservación de espermatozoides es una parte esencial de los programas de mejoramiento genético y selección de reproductores (Barbas et al., 2009). El uso de nuevos dilutores es fundamental para la supervivencia de espermatozoides sometidos a procesos físicos como la criopreservación. Los espermatozoides de equinos son extremadamente sensibles a las alteraciones celulares generadas por la congelación, de allí que los dilutores disminuyen el punto de fusión de las soluciones, reduciendo la concentración intra y extra celular de solutos, evitando el daño celular (Palma, 2001).

En la composición de muchos dilutores se encuentra el EDTA (Martin et al., 1979), el cual está compuesto a base de azúcares (lactosa), sales, albúmina y lípidos, garantizando la disminución del shock térmico y la formación de cristales de hielo intracelular, evitando la lisis celular poscongelación. Asimismo, el uso de carbohidratos, especialmente de lactosa, proporciona la energía necesaria para el metabolismo celular del espermatozoide. Bruemmer (2006) demostró un aumento de la motilidad espermática en el equino al utilizar como dilutor el medio Lactosa-EDTA.

La adición de plasma seminal equino es otro elemento que puede ayudar a aumentar la viabilidad de los espermatozoides epididimarios después del congelamiento (Troedson et al., 2002). Por consiguiente, el presente estudio fue diseñado con la finalidad de evaluar el efecto del dilutor lactosaEDTA-plasma seminal en la viabilidad de espermatozoides epididimarios de equino poscongelación. 


\section{Materiales y Métodos}

\section{Material Biológico}

Se utilizaron 12 pares de testículos de potros criollos adultos, con edades entre $10 \mathrm{a}$ 16 años, beneficiados para consumo en el camal «Casa Blanca», en Pachacamac, Lima, Perú. Los testículos se colectaron inmediatamente después del beneficio, retirándose la túnica vaginal visceral y lavándose con suero fisiológico. Se colocaron en tubos cónicos de $50 \mathrm{ml}$ de polietieleno $\left(\right.$ Falcon $\left.{ }^{\circledR}\right)$ conteniendo $10 \mathrm{ml}$ de solución fisiológica salina al $0.9 \%$ con antibióticos (100 UI/ml penicilina y 100 $\mathrm{mg} / \mathrm{ml}$ estreptomicina).

Los testículos fueron transportados en refrigeración $\left(5^{\circ} \mathrm{C}\right)$ al Laboratorio de Biotecnología y Fisiología Animal de la Facultad de Ciencias Biológicas, Universidad Ricardo Palma, Lima, en un lapso no mayor de dos horas. El estudio se realizó durante los meses de abril a julio de 2010.

\section{Espermatozoides Epididimarios}

Los testículos fueron lavados cinco veces con una solución buferada (PBS) a $37{ }^{\circ} \mathrm{C}$, secándolos con papel absorbente. Se separaron los epidídimos y el conducto deferente mediante un corte con tijera recta.

Se hicieron varios cortes transversales en la cola epididimaria con la finalidad de facilitar la salida de los espermatozoides. El lavado retrogrado se hizo insertando una aguja de $18 \mathrm{G}$ en la entrada del conducto deferente (Cary et al., 2004; Heise et al., 2011) con 10 $\mathrm{ml}$ de diluyente lactosa-EDTA sin yema de huevo. Luego se introdujo $5 \mathrm{ml}$ de aire en el conducto deferente para terminar de evacuar el contenido. Los espermatozoides recuperados fueron recepcionados en placas (Falcón ${ }^{\circledR}$ 35-3001) estériles.
Se colocó $1 \mathrm{ml}$ de la muestra epididimaria en tubos Eppendorf® ( $2 \mathrm{ml})$, en baño María a $37^{\circ} \mathrm{C}$ por 5 minutos para la evaluación microscópica.

\section{Preparación del Dilutor}

Se utilizó el dilutor descrito para la congelación de espermatozoides equinos (Devireddy et al., 2002). La fracción A fue en base a lactosa (11 g), EDTA( $0.1 \mathrm{~g})$, citrato de sodio $(0.089 \mathrm{~g})$, bicarbonato de sodio $(0.008 \mathrm{~g})$ y yema de huevo $(10 \mathrm{ml})$. La fracción B se preparó con $6 \mathrm{ml}$ de glicerol completándose hasta $100 \mathrm{ml}$ con agua bidestilada.

\section{Preparación del Plasma Seminal Equino}

El plasma seminal fue obtenido del eyaculado de dos sementales de la raza $\mathrm{Ca}$ ballo Peruano de Paso, de un haras en Mamacona, Lima. El semen colectado fue centrifugado a $900 \mathrm{~g}$ durante $9 \mathrm{~min}$.

Volúmenes iguales de plasma seminal de los dos sementales se combinaron y el plasma seminal agrupado se centrifugó a 27 $000 \mathrm{~g}$ (Allegra ${ }^{\circledR} \mathrm{X}-30$ Series) por $40 \mathrm{~min}$, con la finalidad de eliminar cualquier resto de espermatozoides.

\section{Fase Experimental}

Las muestras con motilidad mayor de $30 \%$ fueron procesadas y criopreservadas utilizando una dilución de 1:1 con el dilutor, obteniendo una concentración final de 386.3 x $10^{6}$ espermatozoides $/ \mathrm{ml}$. Se tuvo un periodo de equilibrio de 2 horas a $4{ }^{\circ} \mathrm{C}$ y luego el semen diluido fue colocado en pajillas de $0.5 \mathrm{ml}$ (IVM Technologies, Francia) y selladas con alcohol de polivinil. Las pajillas fueron expuestas a vapores de nitrógeno líquido a $4 \mathrm{~cm}$ del nivel de nitrógeno por $10 \mathrm{~min}$ y luego fueron sumergidas en el nitrógeno líquido $\left(-196^{\circ} \mathrm{C}\right)$ por 10 días (Monteiro et al., 2011). 
Para la descongelación, la pajilla se sumergió en agua por $30 \mathrm{~s} \mathrm{a} 37^{\circ} \mathrm{C}$ (Cary et al., 2004; Salazar et al., 2011), evaluándose la motilidad progresiva, viabilidad, morfología e integridad funcional de la membrana (HOS). Para esto, se siguieron los siguientes procedimientos:

- Motilidad progresiva bajo un objetivo de $10 \mathrm{X}$ de un microscopio (CME Leyca $\left.{ }^{\circledR}\right)$.

- Viabilidad, mediante coloración eosinanigrosina

- Morfología, utilizando la batería de Diffquick ${ }^{\circledR}$, observándose con los objetivos de $40 \mathrm{X}$ y $100 \mathrm{X}$.

- Integridad funcional de la membrana plasmática (HOS). A $10 \mu \mathrm{l}$ de la muestra se le agregó $1 \mathrm{ml}$ de solución hiposmótica (citrato de sodio $7.35 \mathrm{~g} / 1$; fructosa $13.51 \mathrm{~g} / 1$; a pH 7.2), e incubada a $37{ }^{\circ} \mathrm{C}$ por $30 \mathrm{~min}$ (Jeyendram et al., 1984). En cada parámetro se contaron 100 espermatozoides (Vidament et al., 2002; Sieme et al., 2003; Heise et al., 2011).

- La concentración espermática se hizo por recuento en una cámara de Neubauer (James, 2004).

\section{Análisis Estadístico}

Se evaluaron las características de los espermatozoides en fresco y posterior a la descongelación mediante la prueba de análisis de varianza y «t» Student con un grado de significación de $\mathrm{p}<0.05$ (Álvarez, 2007). En el análisis estadístico se empleó el programa SPSS v. 21.0.

\section{Resultados y Discusión}

La motilidad progresiva de espermatozoides epididimarios fue de $43.3 \%$ (Cuadro 1), siendo menor del 57\% reportado por James (2004). No obstante, Heise et al. (2011) obtienen un amplio rango (de 10 a 75\%) en epidídimos de cuatro sementales poscastración. Por otro lado, la motilidad posdencogelación fue significativamente menor $(16.3 \%, \mathrm{p}<0.05)$, en tanto que Heise et al. (2011) reportan entre 5 a $10 \%$ y James (2004) y Monteiro et al. (2011) obtienen 46 y $36.2 \%$, respectivamente. En el presente estudio, los espermatozoides estuvieron expuestos al dilutor lactosa-EDTA-plasma seminal por $2 \mathrm{~h}$, en tanto que James (2004) añade glicerol en fracciones de $0.125 \%$ del volumen total en $30 \mathrm{~min}$, llegando al volumen apropiado en $2 \mathrm{~h}$.

La motilidad progresiva posdescongelación en el equino debe ser superior al $30 \%$ para obtener resultados aceptables de concepción (Samper y Morris, 1998). El valor de $16.4 \%$ del presente estudio quedó por debajo de las motilidades obtenidas y recomendadas por Schulman et al. (2003) de 29 a $40 \%$ y de Hernández et al. (2012) de 26 a 53\% para su uso en programas de IA.

Cuadro 1. Características del esperma obtenido de colas de epidídimos de equinos antes y después de la congelación, utilizando un dilutor a base de lactosa-EDTA y plasma seminal

\begin{tabular}{lccccc}
\hline Semen & $\begin{array}{c}\text { Motilidad } \\
\text { progresiva } \\
(\%)\end{array}$ & $\begin{array}{c}\text { Viabilidad } \\
(\%)\end{array}$ & $\begin{array}{c}\text { Morfología } \\
\text { normal } \\
(\%)\end{array}$ & $\begin{array}{c}\text { Concentración } \\
\text { espermática } \\
(\mathrm{x} \mathrm{10} / \mathrm{ml})\end{array}$ & $\begin{array}{c}\text { HOS } \\
(\%)\end{array}$ \\
\hline Fresco & $43.3 \pm 11.5^{\mathrm{a}}$ & $48.3 \pm 9.5^{\mathrm{a}}$ & $67.1 \pm 9.5^{\mathrm{a}}$ & $368.3 \pm 245.9$ & $48.3 \pm 8.3^{\mathrm{a}}$ \\
Descongelado & $16.4 \pm 10.8^{\mathrm{b}}$ & $40.5 \pm 7.5^{\mathrm{a}}$ & $56.5 \pm 11.4^{\mathrm{b}}$ & & $45.5 \pm 10.8^{\mathrm{a}}$ \\
\hline
\end{tabular}

${ }^{a, b}$ Superíndices diferentes dentro de columnas indica diferencia significativa $(p<0.05)$ 
El porcentaje de espermatozoides vivos fue $48.3 \%$, valor inferior al $91 \%$ reportado por Heise et al. (2011) en muestras de epidídimo. La diferencia puede estar asociada a la edad de los animales, ya que las muestras corresponden a caballos con más de 10 años, mientras que en el otro estudio se utilizaron sementales de 4 a 5 años de edad, obtenidas después de la castración. No obstante, la viabilidad de los espermatozoides no se vio afectada por la congelación; posiblemente por el efecto benéfico de la yema de huevo, la cual aporta grasas saturadas, protegiendo a las células contra el shock osmótico (Crabo, 2001). Asimismo, el plasma seminal podría estar ejerciendo un efecto protector.

El porcentaje de espermatozoides normales fue de 67.1 y 56.5 en las muestras pre y poscongelación, respectivamente; valores superiores al 35.4 y $29.9 \%$ reportados por Heise et al. (2011). Las frecuencia de anormalidades encontradas en cabeza, cuello y cola fueron de $14.6,5.8$ y $12.6 \%$ en las muestras frescas, coincidiendo con los resultados de Heise et al. (2011).

\section{ConClusiones}

- La técnica de lavado retrogrado, con el uso del diluyente lactosa-EDTA, permitió recuperar espermatozoides epididimarios viables en equinos.

- La criopreservación de espermatozoides epidimarios de equino, utilizando el dilutor lactosa-EDTA suplementada con plasma seminal equino, afectó negativamente la motilidad posdescongelación.

\section{Agradecimientos}

Los autores agradecen al Programa de Bioemprendimiento y Biocluster Empresarial de la Facultad de Ciencias Biológicas, Universidad Ricardo Palma, por la conexión con el haras. Asimismo, a los integrantes del Laboratorio de Biotecnología y Fisiología Animal, por el apoyo en la realización del trabajo.

\section{Literatura Citada}

1. Alvarez R. 2007. Estadística aplicada a las ciencias de la salud. España: Ed Díaz de Santos. 1030 p.

2. Armas S, Fernández V, Vásquez M, Santiani A. 2011. Determinación del tiempo máximo para recuperar y criopreservar espermatozoides obtenidos de la cola del epidídimo en caninos post orquiectomía. Rev Inv Vet Perú 22: 199-205.

3. Barbas JP, Mascarenhas RD. 2009. Cryopreservation of domestic animal sperm cells. Cell Tissue Bank 10: 49-62. doi: 10.1007/s10561-008-9081-4

4. Bruemmer JE. 2006. Collection and freezing of epididymal stallion sperm. Vet Clin North Am Equine Pract 22: 677-682. doi: 10.1016/j.cveq.2006.08.007

5. Cary JA, Madill S, Farnsworth K, Hayna JT, Duoos L, Fahning ML. 2004. A comparison of electroejaculated and epididymal sperm collection techniques in stallions. Can Vet J 45: 35-41.

6. Crabo BG. 2001. Physiological aspects of stallion semen cryopreservation. In: Proc Annual Convention of the American Association of Equine Practitioners. San Diego, California, USA: AAEP.

7. Devireddy RV, Swanlund DJ, Olin T, Vincente $W$, Troedsson MH, Bischof JC, Roberts KP. 2002. Cryopreservation of equine sperm: optimal cooling rates in the presence and absence of cryoprotective agents determined using differential scanning calorimetry. Biol Reprod 66: 222-231. doi: 10.1095/ biolreprod66.1.222

8. Heise A, Thompson PN, Gerber D. 2011. Influence of seminal plasma on fresh and post-thaw parameters of stallion epididymal spermatozoa. Anim Reprod Sci 123: 192-201. doi: 10.1016/ j.anireprosci.2010.11.017

9. Hernández PJE, Fernández RF, Rodríguez SJ, Soto MY, Verona JE, García $R A$. 2012. Post-thaw acrosomal viability and reaction in sperm obtained from equine epididymis tail. Rev Salud Anim 34: 84-88. 
10. James AN. 2004. Preservation of sperm harvested from the rat, caprine, equine and bovine epididymis. PhD Thesis. USA: Faculty of the Louisiana State University and Agricultural and Mechanical College, Baton Rouge. 229 p.

11. Jeyendran RS, Van Der Ven H, PerezPelaez M, Crabo BG, Zaneveld LJ. 1984. Development of an assay to assess the functional integrity of human sperm membrane and its relationship to other semen characteristics. J Reprod Fertil 70: 219-228. doi: 10.1530/ jrf.0.0700219

12. Martin JC, Klug E, Günzel AR. 1979. Centrifugation of stallion semen and its storage in large volume straws. J Reprod Fertil 27: 47-51.

13. Monteiro GA, Papa FO, Zahn FS, Dell'Aqua Jr JA, Melo CM. Maziero RR, Avanzi BR, et al. 2011. Cryopreservation and fertility of ejaculated and epididymal stallion sperm. Anim Reprod Sci: 127: 197-201. doi: 10.1016/j.anireprosci.2011.08.002

14. Palma GA. 2001. Biotecnología de la reproducción. Argentina: Ed Instituto Nacional de Tecnología Agropecuaria. $704 \mathrm{p}$.

15. Papa FO, Melo CM, Fioratti EG, Dell'Aqua Jr JA, Zahn FS, Alvarenga $M A .2008$. Freezing of stallion epidymal sperm. Anim Reprod Sci 107: 293-301. doi: 10.1016/j.anireprosci.2008.05.003

16. Salazar Jr JL, Teague SR, Love CC, Brinsko SP, Blanchard TL, Varner
DD. 2011. Effect of cryopreservation protocol on post-thaw characteristics of stallion sperm. Theriogenology 76: 409418. doi: 10.1016/j.theriogenology.2011.02.016

17. Samper JC, Morris CA. 1998. Current methods for stallion semen cryopreservation: a survey. Theriogenology 49: 895903. doi: 10.1016/S0093-691X(98)00039-9

18. Schulman ML, Gerber D, Nurton J, Guthrie AJ, Joubert K, Volkmann DH. 2003. Effects of halothane anaesthesia on the cryopreservation of epididymal spermatozoa in pony stallions. Equine Vet J 35: 93-95. doi: 10.2746/042516403775467333

19. Sieme H, Martinsson G, Rauterberg H, Walter K, Aurich C, Petzoldt R., Klug E. 2003. Application of techniques for sperm selection in fresh and frozenthawed stallion semen. Reprod Domest Anim 38: 134-140. doi: 10.1046/j.14390531.2003.00416.x

20. Troedsson MHT, Alghamdi AS, Mattisen J. 2002. Equine seminal plasma protects the fertility of spermatozoa in an inflamed enviroment. Theriogenology 58: 453-456. doi: 10.1016/S0093691X(02)00862-2

21. Vidament M, Daire C, Yvon JM, Doligez P, Bruneau B, Magistrini M, Ecot P. 2002. Motility and feritily of stallion semen frozen with glycerol and/ or dimethyl formamide. Theriogenology 58: 249-251. doi: 10.1016/S0093691X(02)00854-3 